\title{
DEVELOPMENT AND PERFORMANCE EVALUATION OF LOCALIZATION ALGORITHM FOR VARIETY OF TOPOLOGIES IN WIRELESS SENSOR NETWORKS
}

\author{
Anil A. Agashe ${ }^{1}$, R. S. Patil ${ }^{2}$ \\ ${ }^{\text {I}}$ Electronics Engineering Dept. WCE, Sangli, Maharashtra, India, \\ ${ }^{2}$ Electronics Engineering Dept. D.Y. Patil COE, Kolhapur, Maharashtra, India
}

\begin{abstract}
Wireless sensor networks have emerged from military needs and found its way into civil applications. Today wireless sensor networks have become a key technology for different kinds of smart environments. Sensor node localization which is determining where a given sensor node is physically or relatively located is extremely crucial for most of the applications in wireless sensor networks. The procedure through which the sensor nodes obtain their positions is called localization. Many localization algorithms have been proposed for wireless sensor networks. In this article, we describe our newly developed localization algorithm and performance evaluation of this localization algorithm with square, ' $C$ ' and ' $L$ ' shape network topology.
\end{abstract}

Keywords- sensor network, localization algorithms

\section{INTRODUCTION}

The wireless field has been experiencing exponential growth in the past decade. We have seen great advances in network infrastructures, rapid growth of cellular network users, the growing availability of wireless applications, and the emergence of omnipresent wireless devices. The mobile devices are becoming smaller, cheaper, more convenient, and more powerful. In addition to that of the traditional cellular networks, an exponential growth of the wireless device that connects wireless communication devices together to create a wireless network is also being experienced. Many wireless devices can be connected together to create larger network, which is ad hoc network. A wireless ad hoc network is a collection of autonomous nodes or terminals that communicate with each other by forming a multi hop radio network and maintaining connectivity in a decentralized manner. The wireless nodes communicate over wireless links; thus, they have to compete with the effects of radio communication, such as noise, fading, and interference. In addition, the links typically have less bandwidth than in a wired network. Each node in a wireless ad hoc network functions as both a host and a router, and the control of the network is distributed among the nodes. Lowcost, easily installed access points grew rapidly in popularity in the late 1990s and early 2000s. They can also run more applications on the network services. Ad hoc wireless networks, in which a group of potentially mobile units equipped with radio transceivers, can communicate without any fixed infrastructure. Miniaturization of semiconductor technology has lead to the development of small, low power and inexpensive sensor devices often called as nodes. Network of such sensor devices is nothing but wireless sensor network. A wireless sensor network is a network made up of hundreds or thousands of devices using sensors to monitor different conditions, such as temperature, sound, vibration, pressure, motion, or pollutants, at different locations. Usually these devices are small and inexpensive, so that they can be produced and deployed in large numbers. Nodes of such networks can be positioned, randomly or following a predetermined scheme, over a given area; can both collect data and, at the same time, act as communication centers.

Self-localization capability is a highly desirable characteristic of sensor network. In environmental monitoring applications, water quality monitoring and precision agriculture, the measurement data are meaningless without knowing the location from where the data are obtained. Moreover, location estimation may enable a number of applications such as inventory management, intrusion detection, road traffic monitoring, health monitoring, investigation and surveillance.

\section{THE COMPONENTS OF LOCALIZATION SYSTEM}

Localization systems can be divided into three different components:

- Distance and/or angle estimation: This component is accountable for estimating information about the distances and/or angles between two nodes. This information will be used by the other components of the localization system. 
- Location computation: This component is responsible for computing a node's position based on available information concerning distances/ angles and positions of reference nodes.

- Localization algorithm: This is the main component of a localization system. It determines how the available information will be manipulated in order to allow most or all of the nodes of a WSN to estimate their positions.

Various methods are used to get distance and/or angle estimation. These methods include received signal strength indication (RSSI), time of arrival/time difference of arrival (ToA/TDoA), angle of arrival (AoA), and communication range.

\section{SYSTEM MODEL}

For most applications, sensed data without spatial and temporal coordinates is of very limited use. Sensor nodes have to be aware of their location to be able to specify "where" a certain event takes place. Therefore, the problem of localizing the sensors is of paramount importance for many classes of sensor network applications. Determining the physical location of the sensors after they have been deployed is known as the problem of localization.

The system model considered here is a wireless sensor network which is composed of $\mathrm{n}$ nodes with a communication range of $r$, distributed in a two dimensional sensor field. It has been considered that communication links are symmetric; that is, for any two nodes $u$ and $v$, $u$ reaches $v$ if and only if $v$ reaches $u$ and with the same signal strength $w$.

We have deployed the nodes in Square area, C shape area and L shape area. These deployed nodes can be divided into two categories: Anchors and unknown nodes. Anchors are the static nodes with well-defined coordinates. They constitute a customized ad-hoc network for wireless data communication. Unknown nodes, on the other hand, are distributed at a random. While an unknown node falls in the signal coverage of a specific static node, they communicate with each other by making wireless links. Geometry of fixed anchors for above said three topologies is as shown by Figure1, Figure 2 and Figure 3. The symbols used to show Anchor Node and Unknown node is as follows
Anchor Nodes $\diamond$ Unknown Nodes

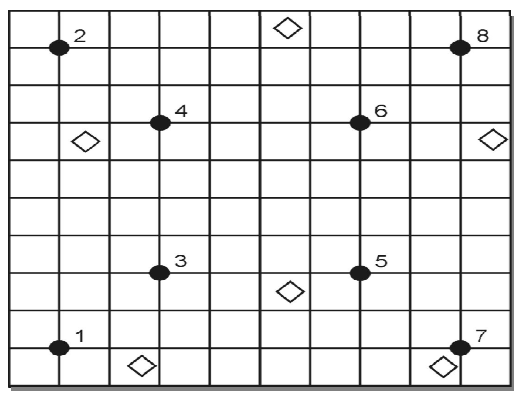

Fig1 Deployment of anchors and unknown nodes in Square topology

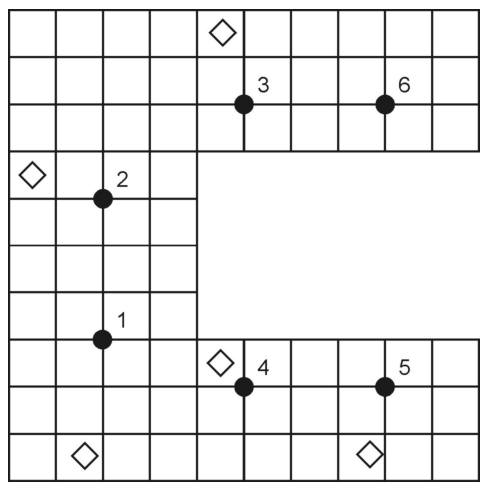

Fig2 Deployment of anchors and unknown nodes in $\mathrm{C}$ shape topology

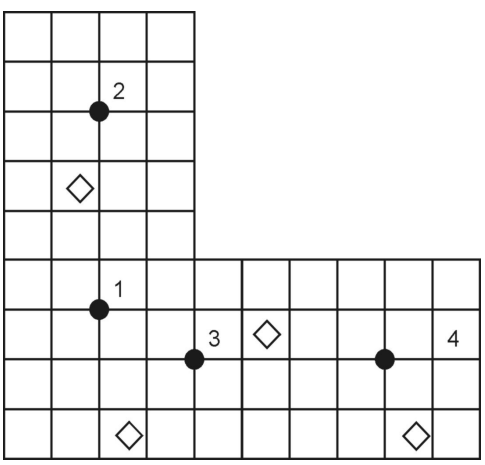

Fig3 Deployment of anchors and unknown nodes in L shape topology

Anchor node refers to the nodes having predefined locations. They are also known as landmarks, these are the nodes that do not need a localization system in order to estimate their physical positions. Their localization is obtained by manual placement or external means such as GPS. These nodes form the base of localization systems for WSNs. Unknown nodes refers to the nodes of the network that do not know their localization 
information. To estimate their positions is the main goal of a localization system.

Ad hoc anchor nodes are those nodes which were initially unknown nodes that manage to estimate their positions by using the localization scheme. The number of ad hoc anchor nodes and the normalized localization error of nodes are the main parameters for determining the quality of a localization system.

\section{PROPOSED METHOD OF LOCALIZATION}

The proposed localization method is as follows

1. Initialize the sensor network which is consisting of anchor nodes and unknown nodes.

2. Select the required topology from Square, C and L shape topology.

3. Designate anchor nodes as fixed anchor nodes and place the fixed anchor nodes according to particular geometry.

4. The distances of the unknown node from all the anchor nodes are calculated.

5. Choose the three anchor nodes which are nearest to unknown node to form a triangle. The centroid of triangle is determined which is the position of unknown node.

6. If unknown node does not lie in the range of three anchor nodes, select two anchor nodes, which are nearest to unknown node.

7. If the unknown node lies in the range of two anchor nodes its estimated position is calculated as centre of the line joining the points of intersection of the two circles whose centres are the anchor nodes and radii are the radio range of these anchors.

8. Once an unknown node gets localized, designate this node as candidate ad hoc anchor node. This node is now available as ad hoc anchor node.

9. This ad hoc anchor node is now available to work as anchor node for localization of remaining unknown nodes.

10. Steps 4 to 9 will be repeated until all unknown nodes get localized.

11. The performance of algorithm is measured with following performance measures.

1. Number of Candidate Ad hoc nodes for particular radio range,

2. Percentage of ad hoc anchor nodes and

3. Normalized localization error

Let us consider the scenario as shown in the Figure 4 . It is proposed to find out the location of node denoted as $\mathrm{S}$, whose co ordinates are $(x, y)$.

As a first step the number of fixed anchor nodes lying in the radio range of node $\mathrm{S}$ is determined. From these nodes three nodes are chosen arbitrarily to form a triangle denoted in the
Figure 4 as $\triangle \mathrm{ABC}$. The positions of three known nodes $\mathrm{A}, \mathrm{B}, \mathrm{C}$ are $\left(x_{1}, y_{1}\right),\left(x_{2}, y_{2}\right),\left(x_{3}, y_{3}\right)$ respectively. In order to find out the location of unknown node (beacon) denoted by $S$ the centroid of a triangle $\mathrm{ABC}$ is calculated. The co ordinates of centroid are nothing but location of $\mathrm{S}$. As a second step node $\mathrm{S}$ is designated as candidate ad hoc anchor node for further localization.

Steps 1 and 2 are repeated for all nodes, until no more nodes can be assigned designation as candidate ad hoc anchor.

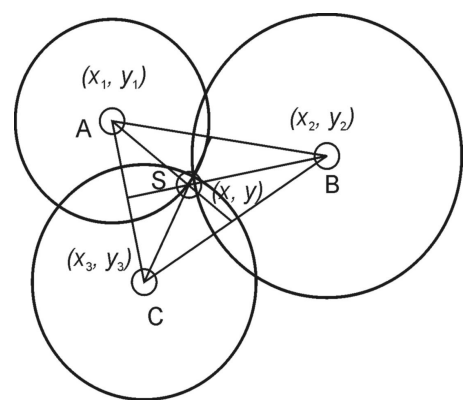

Fig 4: Trilateration paradigm to find location of node that lies in the radio range of three anchors three or more than nodes.

The location of remaining nodes (those who are within range of less than three anchor or ad hoc anchor nodes) is estimated as follows.

If the unknown node lies in the range of two anchor nodes, its estimated position is given as the 'Centre of the line joining the points of intersection of the two circles whose centres are the anchor nodes and radii are the radio range of these anchors'. To find the centre Radical Line Algorithm [5] is used

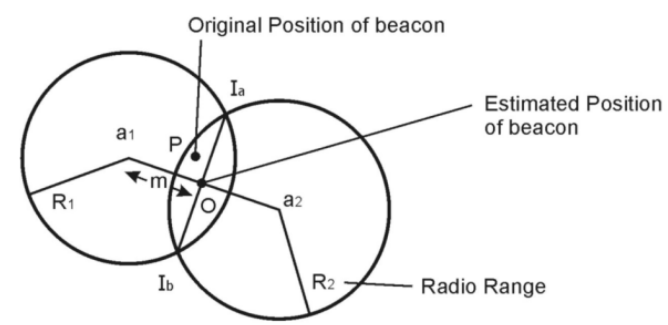

Fig 5 An example of unknown node that lies in the range of two anchor nodes.

\section{SIMULATION MODEL}

A simulation model is developed in MATLAB to study the performance of proposed ad hoc anchor nodes based localization algorithm. Selected deployment region is of 100x100, 200x200, 300x300, 400x400, and 500x500. The anchor nodes are placed at particular location in the deployment 
region. Unknown nodes are distributed at random in the deployment region. Here simulation area considered is Square shape, 'C' shape and ' $L$ ' shape.

For rectangular area eight anchor nodes are placed at certain location which has been determined according to geometry of deployment region. Similarly in ' $C$ ' shape region six anchor nodes and in ' $\mathrm{L}$ ' shape region four anchor nodes are placed at certain locations.

\section{PERFORMANCE MEASURES}

Performance of network is evaluated by calculating following performance measures

A) Candidate Ad hoc Anchor Nodes: During the execution of localization algorithm the unknown nodes which get localized and can work as ad hoc anchor node for localization of remaining unknown nodes are called as Candidate Ad hoc Anchor Nodes.

B) Percentage Ad hoc anchor nodes: During the execution of localization algorithm, the percentage of candidate ad hoc anchor nodes those actually work as ad hoc anchors is calculated. It is desirable to have less percentage of ad hoc anchor nodes used for localization process.

C) Normalized localization error: It is defined as follows. Assume the real coordinate of the unknown node $\mathrm{i}(x, y)$ and its estimated co ordinates are ${ }^{\left(x_{i}, y_{i}\right)}$. The localization error of node $i$ is defined as

$$
e_{i}=\frac{\sqrt{\left(x-x_{i}\right)^{2}+\left(y-y_{i}\right)^{2}}}{\text { Radio Range }}
$$

It is desirable to have a smaller amount of Normalized localization error for ideal localization algorithm.

\section{RESULTS}

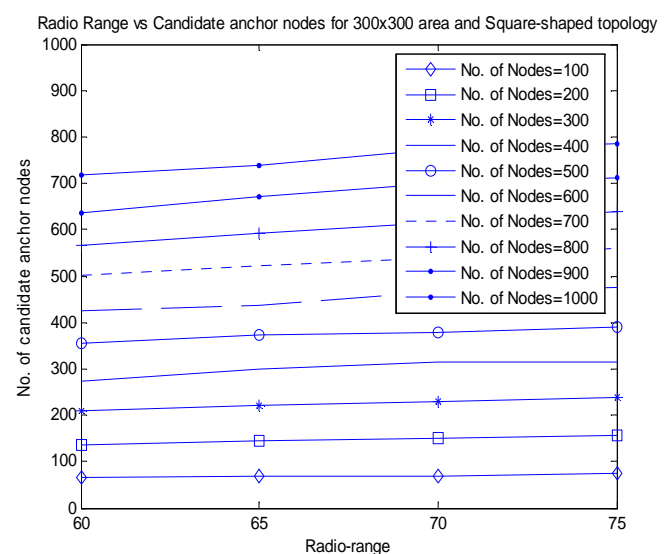

Fig 6 Radio Range Vs Candidate anchor nodes for $300 \times 300$ Square topology
Figure 6 gives candidate anchor nodes generated in the $300 \times 300$ Square topology network at various values of radio ranges from 60 to 75. As there is increase in radio range of sensor nodes, number of candidate ad hoc anchor nodes increases. For the radio range 65 with 200 node density in the network 140 nodes are localized.

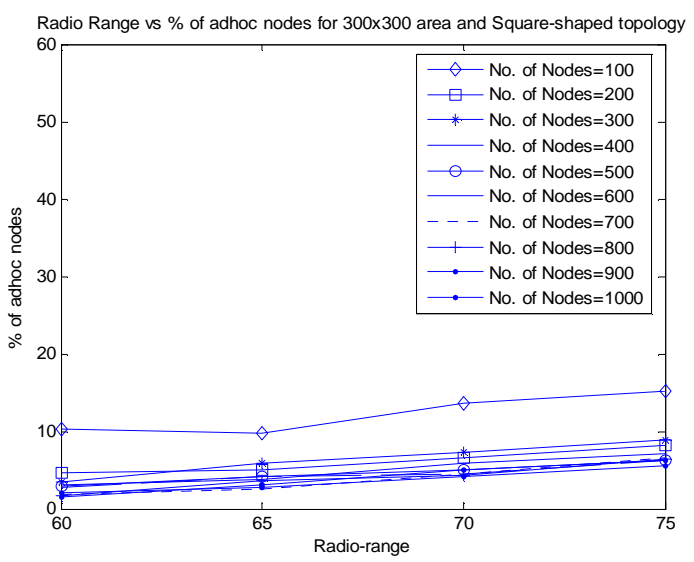

Fig 7 Radio Range Vs \% of ad hoc anchor nodes for $300 \times 300$ Square topology

Figure 7 gives percentage of ad hoc anchor nodes in the $300 \times 300$ Square topology network at various values of radio ranges from 60 to 75 . As radio range increases percentage of ad hoc anchor nodes also increases. For the radio range 70 and node density 100, 13\% nodes works as ad hoc anchor nodes in the network. Here maximum value for percentage of ad hoc anchor nodes is $15 \%$ for radio range 75 .

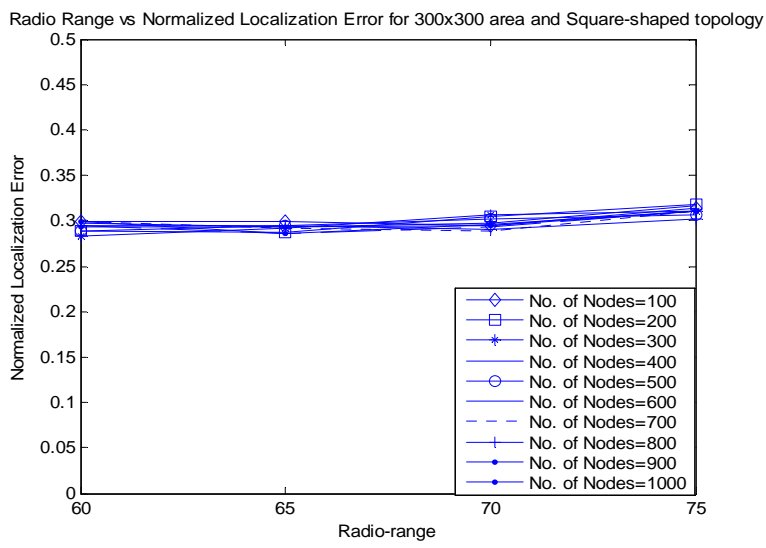

Fig 8 Radio range Vs Normalized Localization error for $300 \times 300$ Square topology

Figure 8 gives Normalized Localization error in the $300 \times 300$ Square topology network at various values of radio ranges from 60 to 75 . In this entire radio range Normalized Localization error changes from 0.3 to 0.32 which is almost constant. 


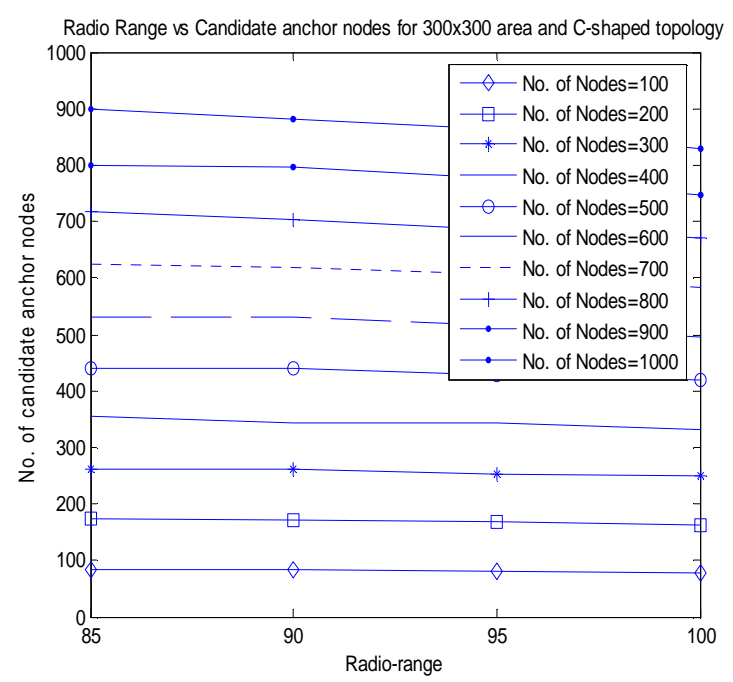

Fig 9 Radio Range Vs Candidate anchor nodes for $300 \times 300$ 'C' shape topology

Figure 9 gives candidate anchor nodes in the $300 \times 300 \mathrm{C}$ topology network at various values of radio ranges from 85 to 100. Here minimum $85 \%$ and maximum $90 \%$ nodes are localized.

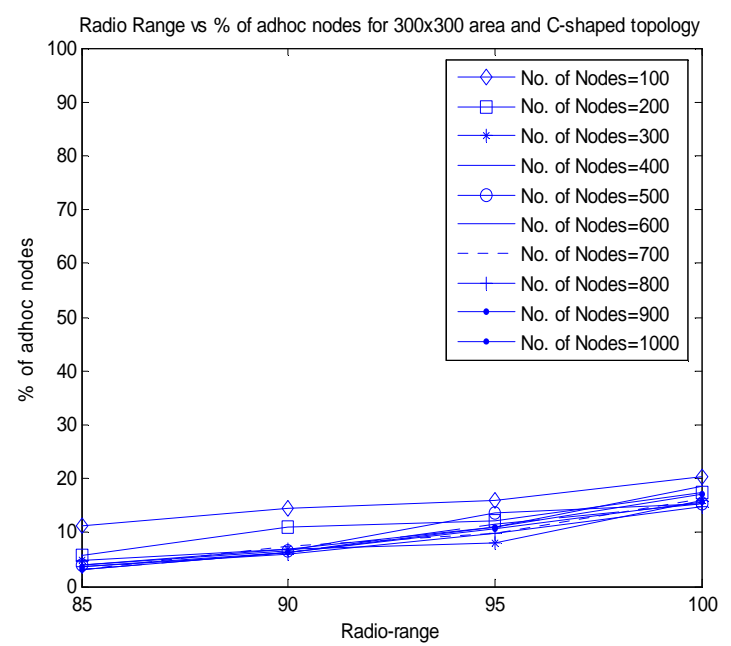

Fig 10 Radio Range Vs \% of ad hoc anchor nodes for $300 \times 300$ 'C' shape topology

Figure 10 shows percentage of ad hoc anchor nodes in the $300 \times 300$ ' $\mathrm{C}$ ' shape topology network at various values of radio ranges from 85 to 100 . As radio range increases percentage of ad hoc anchor nodes increases. For the radio range 100 and node density 100, $20 \%$ nodes works as ad hoc anchor nodes in the network. It demonstrates that for this typical scenario maximum $20 \%$ nodes act as ad hoc anchor nodes.

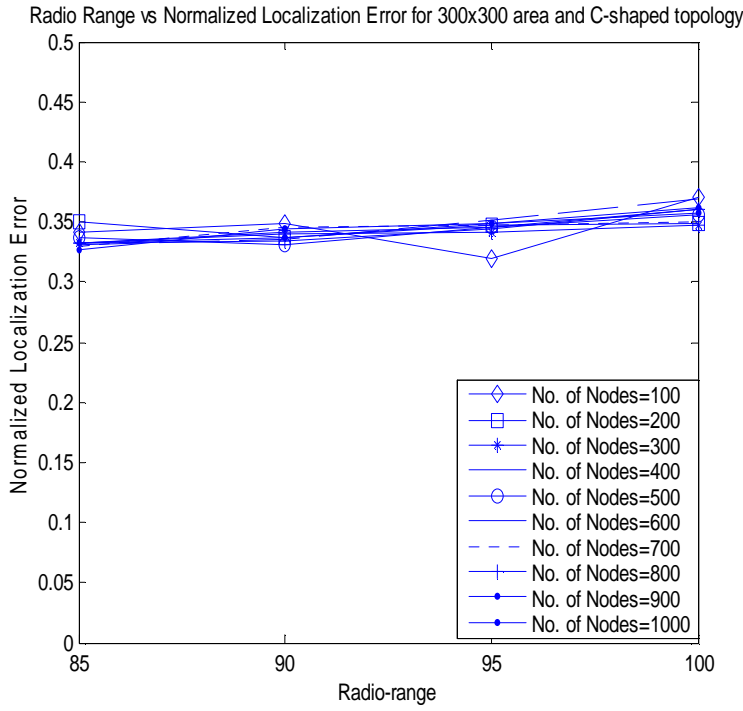

Fig 11 Radio Range Vs Normalized Localization Error for $300 \times 300$ ' $\mathrm{C}$ ' shape topology

Figure 11 gives Normalized Localization error in the $300 \times 300$ $\mathrm{C}$ shape topology network at various values of radio ranges from 85 to 100 . In this entire radio range Normalized Localization Error remains almost constant that is near to 0.35 .

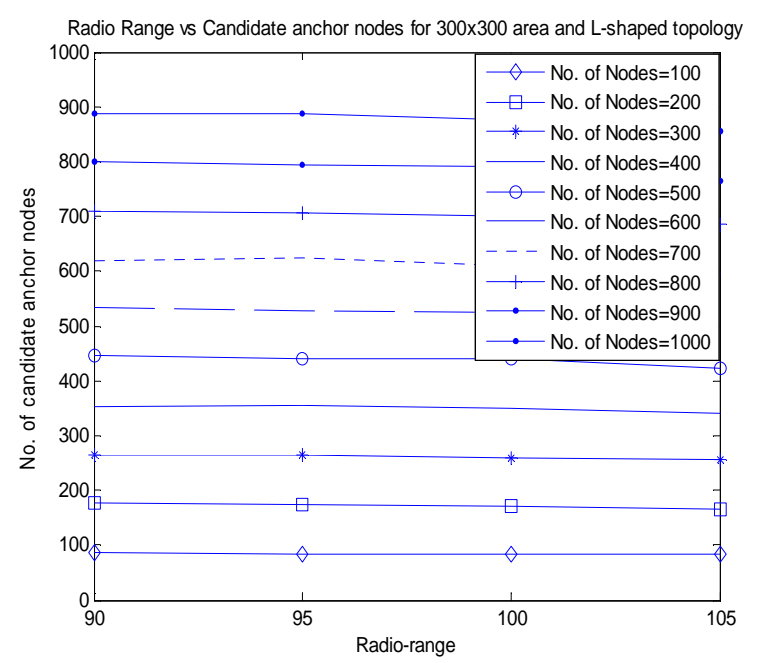

Fig12 Radio Range Vs Candidate anchor nodes for 300×300 'L' shape topology

Figure 12 gives candidate anchor nodes generated in the $300 \times 300 \mathrm{~L}$ topology network at various values of radio ranges from 90 to 105 . It has been observed that even if the radio range of fixed anchor nodes increases, number of localized nodes remains almost constant to $90 \%$. 


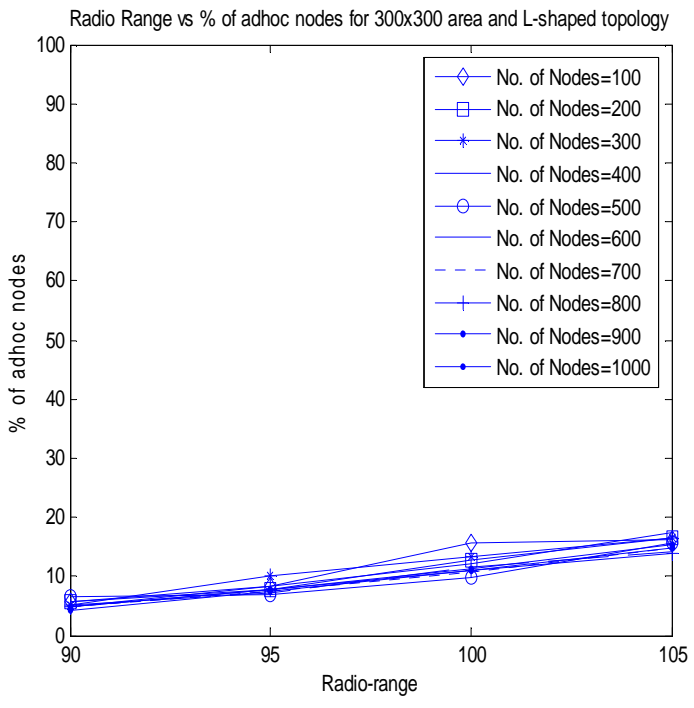

Fig 13 Radio Range Vs \% of ad hoc anchor nodes for $300 \times 300$ ' $L$ ' shape topology

Figure 13 gives percentage of ad hoc anchor nodes in the $300 \times 300$ ' $L$ ' shape topology network at various values of radio ranges from 90 to 105 . As radio range increases percentage of ad hoc anchor nodes also increases up to $20 \%$. For the radio range 100 and node density 100,15\% nodes works as ad hoc anchor nodes in the network.

Figure 14 gives Normalized Localization error in the $300 \times 300 \mathrm{~L}$ shape topology network at various values of radio ranges from 90 to 105 . In this entire radio range Normalized Localization Error remains almost constant. It is 0.34 .

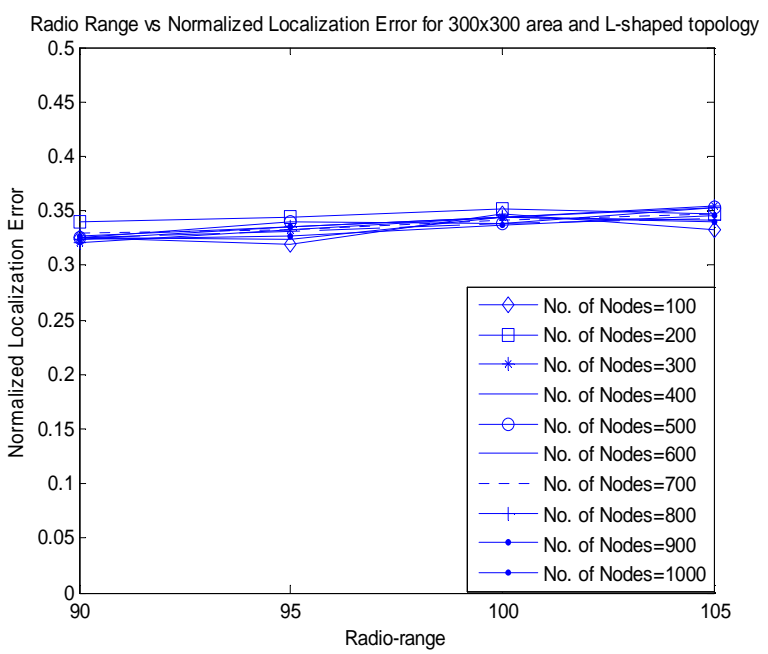

Fig 14 Radio Range Vs Normalized Localization Error for $300 \times 300$ ' $\mathrm{L}$ ' shape topology

\section{CONCLUSIONS}

From the results to obtain better performance of localization algorithm a typical radio range is required to be selected. For Square shape topology radio range required is less compared to "C" \& "L" shape topology. Thus for Square topology less amount of sensor node energy is consumed as radio range required is less. The normalized localization error is less in Square shape topology compared to "C" \& "L" shape topology. The developed algorithm gives less error compared to other range free algorithms. The ad hoc sensor nodes are those nodes which act as anchor nodes in further localization process; once an unknown node is localized it can be used in localization process. The developed localization algorithm distributes the localization task to fixed anchor nodes as well as to ad hoc anchor nodes, which will improve the overall performance of the algorithm.

It has been observed that the percentage of ad hoc anchor nodes required is less in Square topology. Based on a particular localization application the given area is divided first in to Square, then "L" \& "C" shape to get better localization performance. The suggested algorithm gives an insight suggesting typical radio range required for sensor nodes, which type of topology gives better performance, the percentage of ad hoc anchor nodes required depending on no of nodes used in network area.

\section{REFERENCES}

[1]. Eric D. Manley, Huzaifa Al Nahas, and Jitender S. Deogun, "Localization and Tracking in Sensor Systems", Proceedings IEEE International Conference on Sensor Networks, Ubiquitous, and Trustworthy Computing, IEEE Computer Society 2006.

[2]. Salvador Jauregui, Mario Siller, "A big picture on localization algorithms considering sensor logic location",in Proceedings 2009 IEEE International Conference on Systems, Man, and Cybernetics, October 2009. San Antonio, TX, USA, pp. 734-739.

[3]. Anita Panwar, Sh. Ashok Kumar, "Localization Schemes in Wireless Sensor Networks", in Proceedings 2012 Second International Conference on Advanced Computing \& Communication Technologies, IEEE Computer Society, pp. 443- 449.

[4]. D. Niculescu and B. Nath, "Ad-hoc Positioning System", Proceedings Global Telecommunications Conference, IEEE, Volume 5, Nov 25-29, 2001, pp. 2926-2931.

[5]. Hongyang Chen, "Accurate and Energy efficient node localization in wireless sensor networks", University of Tokyo [6]. Chu Bolin, Zheng Zengweillsiwsn, "A new Range-free Localization Algorithm for Large Scale Wireless Sensor Networks", International Conference on Business Computing and Global Information, 2011 pp. 408-411. 